\title{
Avaliação Termoanalítica do Isobutirato Acetato de Sacarose como Antioxidante em Biodiesel
}

\author{
Thermal Analysis Evaluation of Sucrose Acetate Isobutyrate as \\ Antioxidant in Biodiesel Sample
}

\author{
Seme Youssef Reda ${ }^{1}$; Bill Jorge Costa ${ }^{2}$; Renato João Sossela de Freitas ${ }^{3}$; \\ Juan Carlo Villalba ${ }^{4}$; Fauze Jacó Anaissi ${ }^{5}$
}

\begin{abstract}
Resumo
Este trabalho teve como objetivo avaliar por técnica termoanalítica o Isobutirato Acetato de Sacarose (SAIB) como antioxidante em uma amostra de biodiesel etílico obtido de óleo de soja bruto, nas concentrações $0,1000,2000,3000,4000$ e 5000 ppm, para saber se o SAIB poderia ser indicado como um antioxidante em biodiesel. Inicialmente, as propriedades físico-químicas tanto o óleo de soja, quanto do biodiesel foram avaliadas por RMN de hidrogênio-1, que demonstrou que as amostras estavam dentro dos limites de normalidade preconizada pela ANVISA e pela ANP, respectivamente. O SAIB foi testado por TG/DTG/DTA, cujos resultados demonstraram que o mesmo tem relevante resistência térmica e a melhor concentração encontrada de SAIB para a proteção da amostra de biodiesel testada foi $5000 \mathrm{ppm}$. Embora os resultados tenham sido satisfatórios, estudos de resistência oxidativa devem ser realizados para se aprimorar o conhecimento sobre o comportamento do SAIB como antioxidante no biodiesel.
\end{abstract}

Palavras-chave: Isobutirato Acetato de Sacarose. Biodiesel. Análise térmica. SAIB.

\begin{abstract}
This study aimed to evaluate the sucrose acetate isobutyrate (SAIB) as an antioxidant in ethyl biodiesel sample obtained from crude soybean vegetable oil at $0,1000,2000,3000,4000$ and $5000 \mathrm{ppm}$ concentrations, by thermal analysis, to know if the SAIB could be suitable as an antioxidant in biodiesel. Initially, the physicochemical properties of both soybean vegetable oil and the biodiesel were evaluated by ${ }^{1} \mathrm{H}$ NMR which showed that the samples were within normal limits recommended by ANVISA and ANP, respectively. The SAIB has been tested by TG/DTG/DTA, where the results demonstrated that the antioxidant presented significant thermal resistance and the best dosage of SAIB to protect the biodiesel sample tested was $5000 \mathrm{ppm}$. Although the results has been satisfactory, researches of oxidative resistance must to be carried out to improve the knowledge about the SAIB behavior as antioxidant in the biodiesel.
\end{abstract}

Key-words: Sucrose Acetate Isobutyrate. Biodiesel. Thermal analysis. SAIB.

\footnotetext{
1 Farmacêutico Bioquímico, Doutor em Processos Biotecnológicos - UFPR. E-mail: syreda@yahoo.com.br

${ }^{2}$ Diretor Técnico do Instituto de Tecnologia do Paraná - TECPAR.

${ }^{3}$ Professor do Curso de Pós-graduação em Biotecnologia da UFPR.

${ }^{4}$ Doutorando do curso de Pós-Graduação em Química da UNICENTRO.

${ }^{5}$ Professor do curso de Pós-graduação em Química da UNICENTRO.
} 


\section{Introdução}

A utilização de uma fonte de energia autosustentável é uma preocupação mundial e é o foco de muitas pesquisas atualmente. Nesse sentido, o biodiesel tem algumas características importantes que são de interesse global como sua sustentabilidade através do seu ciclo de vida, emitindo muito menos GEEs (Gases do Efeito Estufa) do que os combustíveis convencionais, especificamente (MOTHÉ et al., 2005).

Segundo a Agência Nacional de Petróleo, Gás Natural e Biocombustíveis (2004), alguns parâmetros de qualidade devem ser investigados para que o biodiesel possa ser utilizado em motores a diesel. Dentre os mais importantes, tem-se a estabilidade termo-oxidativa, um parâmetro de relevante significado dentre os prescritos pela ANP para o controle de qualidade do biodiesel, uma vez que esse requisito influi na garantia da sua integridade durante o seu uso (LIANG et al., 2006). $\mathrm{O}$ ataque termo-oxidativo ao biodiesel, resultante do trabalho do motor, influencia propriedades físicas do mesmo, como a viscosidade e o ponto de entupimento de filtro a frio (Biodiesel Analytical Methods, 2008).

Segundo a ANP (2004), qualquer amostra de biodiesel deve suportar uma temperatura de $100^{\circ} \mathrm{C}$ por $6 \mathrm{~h}$ consecutivas, sem se decompor dentro do motor. Como a matéria-prima para a produção de biodiesel nacional é proveniente de oleaginosas, com alto teor de índice de iodo, condicionando as amostras de biodiesel a um elevado teor de insaturações e susceptibilidade termo-oxidativa, faz-se necessário o uso de aditivos ao biodiesel, como a adição de antioxidantes, como forma de proteção ao bicombustível (FERRARI; SOUZA, 2009).

Assim, o objetivo desse trabalho foi testar o Isobutirato Acetato de Sacarose por técnica termoanalítica, para observar se o referido composto poderia ser indicado como um potencial antioxidante no biodiesel, cujo mecanismo de ação anti-oxidativo baseia-se na acepção de elétrons derivados de radicais livres.

\section{Materiais e métodos}

Propriedades físico-químicas do óleo de soja bruto

$\mathrm{O}$ estudo das propriedades físico-químicas do óleo de soja bruto foi realizado por RMN de hidrogênio-1, onde especificamente foram calculados os índices de iodo, de saponificação e de acidez, acidez livre e peso molecular médio, pelo programa PROTEUS RMN H1 (CARNEIRO; REDA; CARNEIRO, 2005) (REDA; CARNEIRO, 2006).

\section{Propriedades físico-químicas do biodiesel}

As propriedades físico-químicas do biodiesel de soja foram realizadas por meio de RMN de hidrogênio-1, em que os índices de iodo e de acidez, teor de glicerina livre e peso molecular médio dos etil-ésteres formados foram calculados pelo programa JV.NH-1 (REDA; COSTA; FREITAS, 2007a), (REDA; COSTA; FREITAS, 2007b), (REDA; COSTA; FREITAS, 2008a), (REDA; COSTA; FREITAS, 2008b).

Análise por ressonância magnética nuclear de hidrogênio

As amostras de óleo ebiodiesel, aproximadamente 10 a 20mg, foram dissolvidas em $0,7 \mathrm{~mL}$ de $\mathrm{CDCl}_{3}$ e seus espectros de RMN foram registrados em espectrômetro Varian, modelo Mercury-300 MHz, operando no modo FT a temperatura ambiente. Para os núcleos de Hidrogênio-1 foram utilizados os seguintes parâmetros de aquisição: pulso: $45^{\circ}$, tempo de relaxação: $1,359 \mathrm{~s}$; tempo de aquisição: 3,64 s; largura de varredura: $4.120 \mathrm{~Hz}$, largura de linha $0,3 \mathrm{~Hz}$. Foram acumuladas 16 repetições para cada decaimento induzido livre (FID). 
Obtenção dos ésteres etílicos por transesterificação dos óleos vegetais

O biodiesel foi produzido por rota etílica, por meio de transesterificação alcalina do óleo de soja bruto com etanol anidro (p.a), em excesso estequiométrico, empregando o hidróxido de potássio $(\mathrm{KOH})$ como catalisador, numa proporção molar etanol: óleo de 6:1 a 0,5\% (m/m) de $\mathrm{KOH}$ à temperatura de reação de $65^{\circ} \mathrm{C}$ por $40 \mathrm{~min}$., sob agitação enérgica constante. Os traços de etanol excedente ao término da reação foram retirados da fase orgânica por evaporação, aumentando-se a temperatura do meio para $80^{\circ} \mathrm{C}$ por $15 \mathrm{~min}$. Após essa fase, adicionou-se hexano para se extrair a fase orgânica. $\mathrm{O}$ hexano foi removido em evaporador rotatório e o biodiesel recuperado foi seco em sulfato de sódio anidro e utilizado para as determinações físico-químicas.

Características fisico-químicas do antioxidante Isobutirato Acetato de Sacarose - SAIB

Foi avaliada a ação protetora do Isobutirato Acetato de Sacarose (SAIB) em uma amostra de biodiesel etílico produzido a partir de uma amostra de óleo de soja bruto, em que quimicamente o antioxidante tem as seguintes características (REDA, no prelo):

Quimicamente, o antioxidante SAIB é $[(2 \mathrm{~S}, 3 \mathrm{~S}, 4 \mathrm{R}, 5 \mathrm{R})-2-$ (acetiloximetil) $-2-$ $[(2 \mathrm{R}, 3 \mathrm{R}, 4 \mathrm{~S} 5 \mathrm{R}, 6 \mathrm{R})-6-$ (acetiloximetil)3,4,5 - tris (2metilpropanoiloxi) oxan - 2 -)il]oxi

$-4-(2-$ metilpropanoiloximetil $)-5-(2$ - metilpropanoiloxiletil)oxolan - 3 - il] 2 metilpropanoato

Fórmula molecular: $\mathrm{C}_{40} \mathrm{H}_{62} \mathrm{O}_{19}$

Massa molecular: $846.909 \mathrm{~g} / \mathrm{mol}$

\section{Análise térmica do antioxidante $S A I B$}

As curvas termogravimétricas e calorimétricas foram obtidas em Analisador Térmico, Seiko Instruments, model 6000, na razão de aquecimento de $10{ }^{\circ} \mathrm{C} \cdot \mathrm{min}^{-1}$, em cadinho de alumina, atmosfera de ar, no intervalo de temperatura de $25-1200^{\circ} \mathrm{C}$, visando verificar o perfil da decomposição térmica (CARRASCO, 1993; WENDHAUSEN, 2006). A seguir foram realizadas análises por TG/DTG/ DTA do biodiesel de soja adicionado de SAIB nas concentrações: 0, 1000, 2000, 3000, 4000 e 5000 ppm, para avaliar a sua estabilidade térmica, sem a adição e com a adição de antioxidante, num intervalo de temperatura de $25-1200^{\circ} \mathrm{C}$.

\section{Resultados e discussão}

Caracterização do óleo de soja bruto.

Do espectro integrado de RMN H1 do óleo vegetal bruto de soja foi possível calcular pelo programa PROTEUS RMN H1, o índice de iodo (II), acidez (A), o índice de saponificação (IS) e o peso molecular médio (PM) dos triacilgliceróis da amostra, conforme demonstrado na Tabela 1.

Tabela 1 - Análise físico-química do óleo de soja bruto calculado pelo programa PROTEUS RMN H1.

\begin{tabular}{|c|c|}
\hline RMN H1 & Resultados \\
\hline I.I. & 118,5 \\
\hline I.S & 195,0 \\
\hline Acidez & 1,5 \\
\hline IA & 2,9 \\
\hline PM & 870,2 \\
\hline
\end{tabular}

Segundo os resultados obtidos, os parâmetros estavam dentro dos preconizados pela ANVISA (1999). Porém, observa-se que o óleo de soja mesmo possuindo um elevado índice de acidez, característico para óleos vegetais brutos, não interferiu com a reação de transesterificação, uma 
vez que ficou no limite de $3,0 \%$, que é o limite para uma reação de transesterificação eficiente (FERRARI; OLIVEIRA; SCABIO, 2005). O óleo de soja também apresentou um elevado índice de iodo (II) o que reflete o grau de insaturação da amostra. Importante dizer sobre esse dado é que o alto grau de insaturação presente na matéria-prima será transferido ao biodiesel, tornando-o igualmente altamente insaturado e assim, susceptível a ataques termo-oxidativos.

\section{Caracterização do biodiesel por RMN H1}

As propriedades físico-químicas do biodiesel foram calculadas pelo programa JV.NH-1 (REDA; COSTA; FREITAS, 2008a), especificamente os índices de iodo (II) e de acidez (IA), teor de glicerina livre (GL) e peso molecular médio (PM) do pool de etil-ésteres de ácidos graxos que compõem o biodiesel. Os resultados obtidos são mostrados na Tabela 2.

Tabela 2 - Propriedades físico-químicas do biodiesel calculadas pelo programa JV.NH-1.

\begin{tabular}{c|c}
\hline RMN H1 & Resultados \\
\hline II & 108.30 \\
IA & 0.55 \\
GL & 0.02 \\
PM & 311.50 \\
\hline
\end{tabular}

Todos os resultados obtidos estão dentro dos limites estabelecidos pela ANP (2004). Contudo, pode-se observar que o biodiesel tem alto índice de iodo, conferido pelo óleo de soja do qual ele se originou, como já exposto.

\section{Análise térmica do antioxidante SAIB}

A curva de TGA/DTA do SAIB (Isobutirato Acetato de Sacarose), na Figura 1, pela análise da curva TG é possível observar a decomposição deste antioxidante, que se inicia próximo de $200^{\circ} \mathrm{C}$, demonstrando sua resistência à ação termo-oxidativa.

Esses dados são confirmados pela curva DTG/ DTA do antioxidante, onde se observa um início de decomposição acima de $200^{\circ} \mathrm{C}$, mostrado pela curva DTA e uma carbonização em torno de $380^{\circ} \mathrm{C}$, demonstrado pelo pico exotérmico da curva DTG.

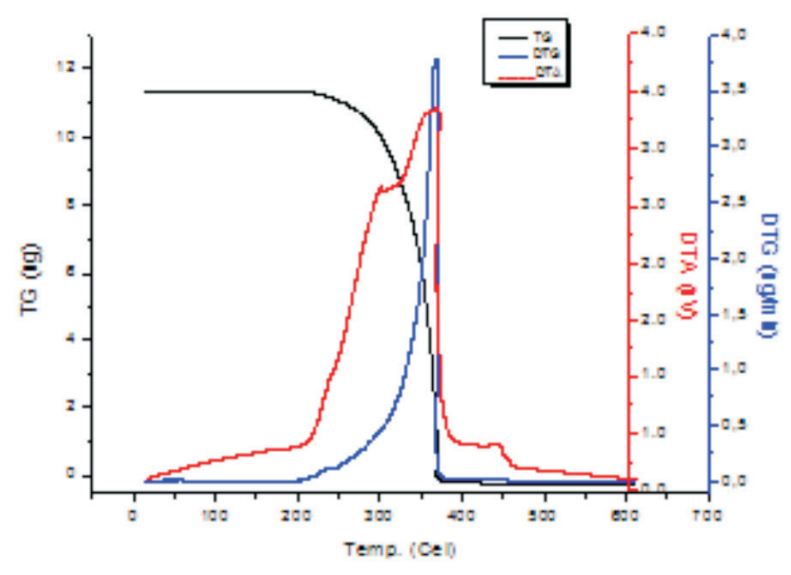

Figura 1. Curva TG/DTG/DTA somente do antioxidante SAIB.

$\mathrm{Na}$ Figura 2 é mostrado o perfil térmico do biodiesel de soja, sem qualquer aditivo químico. Os resultados, demonstrados pela análise da curva TG/DTG do biodiesel mostram que o biodiesel tem uma perda de massa de $96.9 \%$ no intervalo de temperatura entre 25 e $257^{\circ} \mathrm{C}$, com perda total de sua massa em $748^{\circ} \mathrm{C}$. Perfil confirmado pelos padrões comparativos entre as curvas DTG/DTA. Contudo houve a formação de dois picos detectados pela curva DTG em $157^{\circ} \mathrm{C}$ e uma segunda em $172^{\circ} \mathrm{C}$, indicando perda de massa nessas temperaturas. Esse comportamento foi confirmado pela curva TG/ DTA (Figura 3), onde se podem observar dois picos endotérmicos na temperatura acima descrita. Isso pode indicar ocorrência de formação de compostos secundários, na reação térmica do biodiesel no intervalo de temperatura entre 25 e $200^{\circ} \mathrm{C}$, como a formação peróxidos ou hidroperóxidos. Tais compostos podem conduzir à formação de ácidos e aldeídos, ou pode reagir com outra cadeia de ácido graxo formando dímeros e polímeros (CANDEIA et al., 2007). 


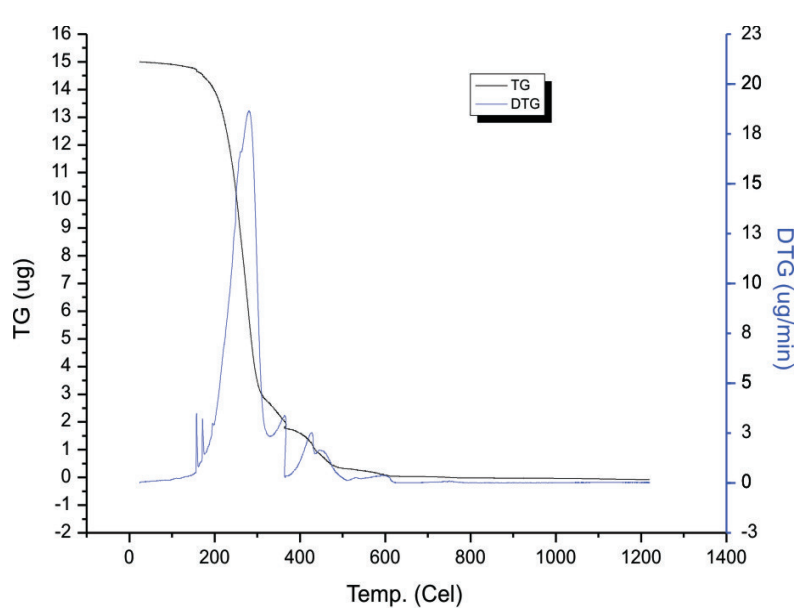

Figura 2. Curva TG/DTG do biodiesel de soja sem a adição de SAIB.

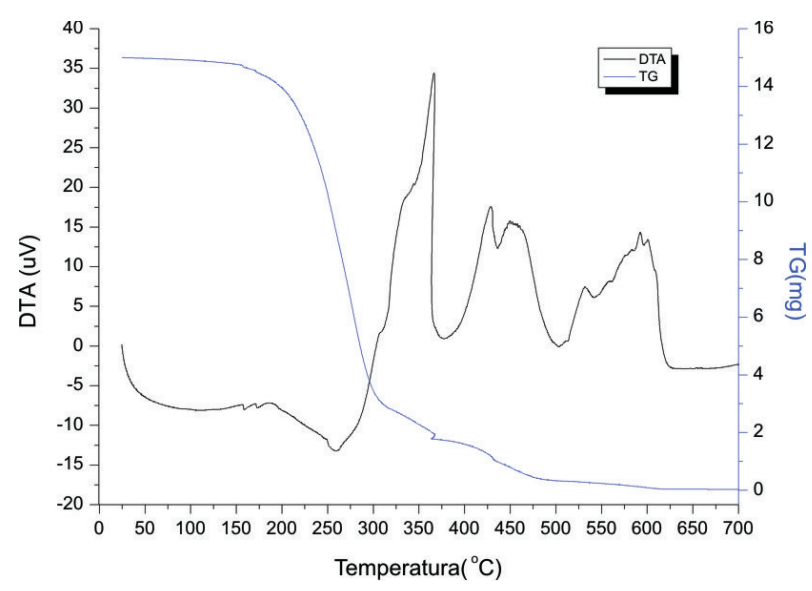

Figura 3. Curva TG/DTA do biodiesel de soja sem a adição de SAIB.

Nas Figuras 2 e 3, pode-se observar que ocorrem varias transições exotérmicas, o qual se pode atribuir a primeira transição, ao processo de oxidação, no qual ocorrem os processos de iniciação, propagação e terminação do biodiesel. Inicialmente, ocorre à polimerização da cadeia de ésteres de ácidos graxos alquílicos com conseqüentemente formação da goma. A temperatura de pico do processo de polimerização para o biodiesel etílico é de aproximadamente $243{ }^{\circ} \mathrm{C}$ e para a combustão da goma e de aproximadamente $450^{\circ} \mathrm{C}$. Logo, os picos endotérmicos demonstrados pela DTA, revelam os processos de volatilização dos componentes do biodiesel e da perda de compostos voláteis formados pelo processo de oxidação do biodiesel. De outro lado, os picos exotérmicos expressam a combustão de polímeros de alto peso molecular durante o processo de análise (OLIVEIRA, 2006).

No estudo do biodiesel de gergelim feito por Almeida et al (2007), observou-se que o biodiesel apresentou uma única etapa de decomposição térmica no intervalo $24-268^{\circ} \mathrm{C}$ com perda de massa de $87,56 \%$. Essa perda foi atribuída à volatilização e/ou decomposição dos ésteres etílicos do biodiesel. Também a curva DTA do biodiesel de gergelim apresentou uma transição endotérmica e pelo menos duas transições exotérmicas significativas. Esse achado foi igualmente encontrado para o biodiesel etílico de soja (Figura 3), que mostrou três transições exotérmicas.

Contudo, nota-se na Figura 3 um pico endotérmico detectado pela curva DTA em $220^{\circ} \mathrm{C}$, porém sem decomposição da amostra apresentada pela curva DTG, o que pode significar um período de transição de primeira ordem, onde existe uma coexistência de fases, em que o parâmetro de ordem exibe um salto. Como a modificação das posições dos átomos que constituem a molécula de biodiesel é contínua, muitas das propriedades da amostra irão variar continuamente. Por isso, em particular, uma transição desse tipo não é acompanhada de liberação de calor (FERRARI; SOUZA, 2009).

Importante notar que a curva DTA apresenta muitas variações exotérmicas nítidas entre 300 e $600^{\circ} \mathrm{C}$, acompanhadas de perda de massa evidenciada pela curva TG. Segundo Rosenhaim (2009), no estudo do biodiesel etílico derivado de óleo de fritura usado analisado por termogravimetria, pode-se observar que as curvas termoanalíticas apresentaram mais de transição, demonstrada pela curva DTA. Esta evidência foi atribuída à combustão de polímeros tanto de baixo peso molecular, como de alto peso molecular formados durante o processo de análise, nas transições exotérmicas entre 280 $600^{\circ} \mathrm{C}$.

Ao observar o comportamento do biodiesel nas diversas concentrações de SAIB, notou-se que à 
medida que a concentração de antioxidante foi sendo aumentada (Figuras 4, 5 e 6) e a perda de massa no intervalo de 25 a $200^{\circ} \mathrm{C}$ foi diminuindo.

O perfil térmico apresentado na Figura 7 (concentração de 4000 ppm) revela perdas mínimas de massa em $102^{\circ} \mathrm{C}$ e em $174^{\circ} \mathrm{C}$, conforme demonstrado pela DTG, sendo este efeito provavelmente atribuído à ação do antioxidante no intervalo de temperatura entre 25 e $200^{\circ} \mathrm{C}$.

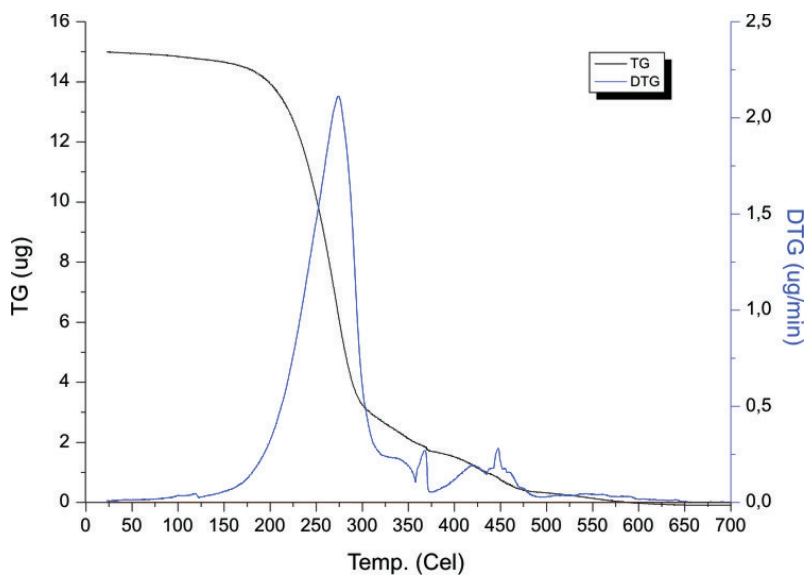

Figura 4. Perfil térmico do biodiesel de soja acrescido do antioxidante SAIB na concentração de 1000 ppm.

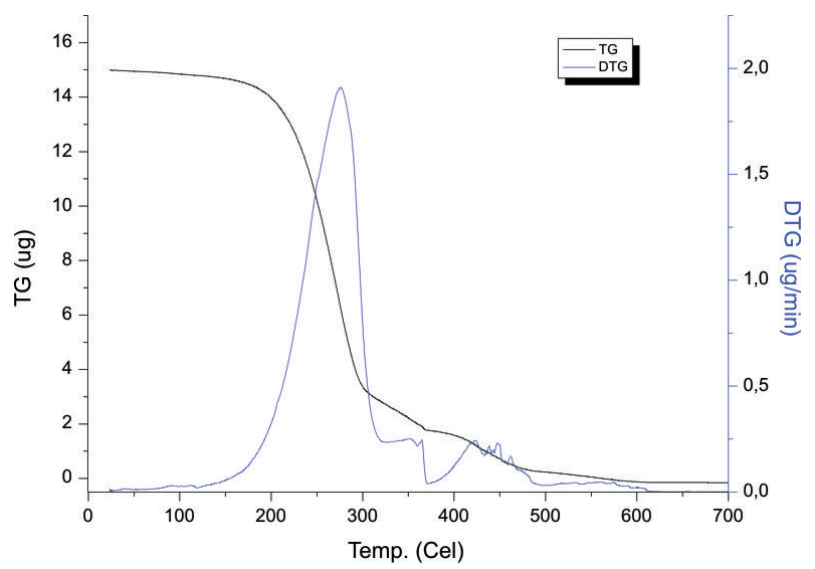

Figura 5. Perfil térmico do biodiesel de soja acrescido do antioxidante SAIB na concentração de 2000 ppm.

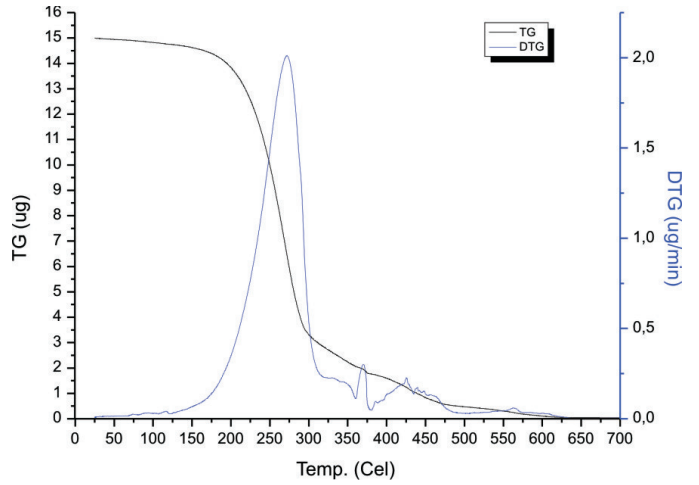

Figura 6. Perfil térmico do biodiesel de soja acrescido do antioxidante SAIB na concentração de 3000 ppm

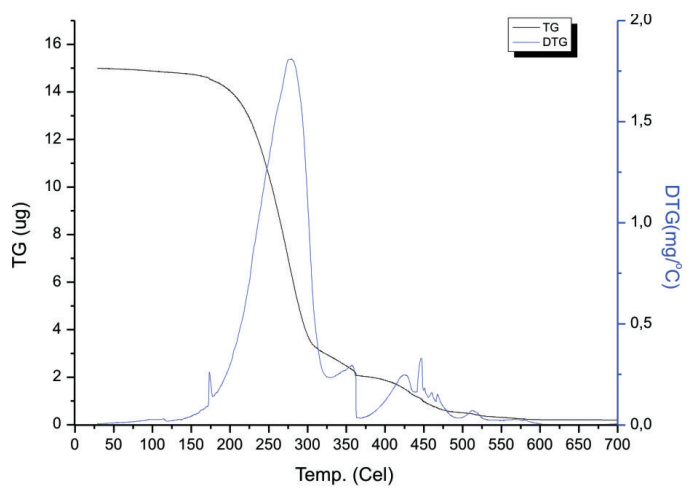

Figura 7. Perfil térmico do biodiesel de soja acrescido do antioxidante SAIB na concentração de 4000 ppm.

Em comparação com a mostra inicial sem antioxidante, percebe-se que o biodiesel acrescido de 5000 ppm de SAIB (Figura 8) pode ser verificado pelo padrão da curva TG/DTG, uma perda mínima de massa, com um pico endotérmico mostrado pela curva TG/DTA (Figura 9).

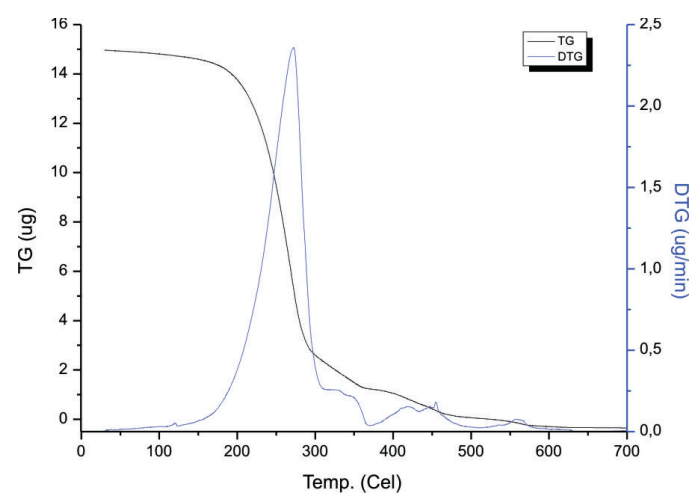

Figura 8. Perfil térmico do biodiesel de soja acrescido do antioxidante SAIB na concentração de 5000 ppm. 


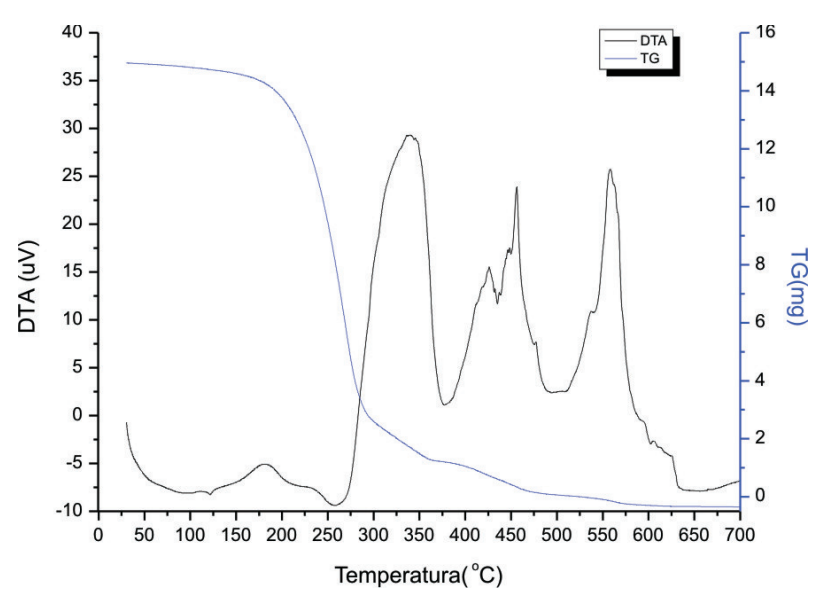

Figura 9. Perfil térmico TG/DTA do biodiesel de soja acrescido do antioxidante SAIB na concentração de 5000 ppm.

Segundo Lima, Carvalho e Santos (2007), a transição endotérmica está em função do processo de absorção de energia devido à reestruturação das moléculas dos ésteres etílicos do biodiesel durante o processo de decomposição e as transições exotérmicas estão relacionadas com a decomposição da amostra registrada pela curva TG do biodiesel de soja. Também se observa pela curva TG/DTG que no intervalo entre 25 e $600^{\circ} \mathrm{C}$ há sucessivas perdas de massa, o que caracteriza a decomposição da amostra pelo processo termo-oxidativo (DANTAS, 2006). Isso está de acordo com o encontrado por Dantas (2006), no estudo termoanalítico do biodiesel obtido do óleo de algodão. Portanto, as transições exotérmicas detectadas representam uma alteração do biodiesel de soja frente ao processo de oxidação, referente à volatilização e/ou decomposição dos ésteres etílicos e as transições endotérmicas podem representar a ocorrência da formação de compostos secundários, na reação de termo-oxidação do biodiesel.

Considerando a faixa de temperatura até $200^{\circ} \mathrm{C}$, observa-se que o grande efeito do uso do SAIB em relação ao biodiesel sem antioxidante está na amostra com teor de 1000 ppm a qual apresentou uma perda de massa em $117^{\circ} \mathrm{C}$, com uma ordem de grandeza menor. A partir daí, este comportamento se repete na amostra com $5000 \mathrm{ppm}$ em $119^{\circ} \mathrm{C}$. $\mathrm{O}$ fato de amostras intermediárias apresentarem comportamentos diversos à medida que o conteúdo de SAIB foi elevado, pode ser atribuído ao grau de pureza das amostras.

\section{Conclusões}

As propriedades físico-químicas tanto do óleo de soja, quanto do biodiesel, avaliados por RMN de hidrogênio-1, demonstraram que as amostras estavam dentro dos limites de normalidade preconizada pela ANVISA e pela ANP, respectivamente. A análise térmica revelou a boa resistência do antioxidante Isobutirato Acetato de Sacarose - SAIB, por meio da curva TG/DTG/ DTA. As análises da ação do SAIB no biodiesel de soja revelaram que a adição de 5000 ppm de antioxidante ao biodiesel proporcionou uma melhor resistência térmica do biodiesel ao aquecimento, no intervalo de temperatura entre $25-200^{\circ} \mathrm{C}$, embora deva ser destacado que, sob o aspecto prático, teores de aditivo acima de 1.000 ppm não seriam factíveis na indústria.

\section{Referencias}

AGÊNCIA NACIONAL DE PETRÓLEO, GÁS NATURAL E BIOCOMBUSTÍVEIS - ANP. Resolução $n^{\circ} 42$, de 24 de Novembro de 2004. Disponível em: $<$ http://www.biodiesel.gov.br/docs/Resolucao_42.pdf $>$. Acesso em: 13 set. 2008.

AGÊNCIANACIONAL DE VIGILÂNCIA SANITÁRIA - ANVISA. Óleo e Gorduras vegetais, 1999. Disponível em: <http://www.anvisa.gov.br/legis/resol/482_99.htm> Acesso em: 13 jun. 2010.

ALMEIDA, K.V.; SOUSA, W.J.B.; SILVA, A.C.; FIRMINO, P.T.; BARROS, A.J.M. Biodiesel. Estudo de Biodiesel Etílico de Gergelim por Termogravimetria e Análise Térmica Diferencial. 2007. Disponível em:< http://www.abq.org.br/biocom/2009/trabalhos/-22-5627. htm> Acesso em: 13 set. 2008.

BIODIESEL ANALYTICAL METHODS. 2008. Disponível em: <http://www.osti.gov/bridge>. Acesso em: 13 set. 2008. 
CANDEIA, R. A.; ROSENHAIM, R.; CONCEIÇÃO, M. M.; SILVA, M. C. D.; SANTOS, I. M G.; SOUZA, A. G.; CAVALCANTI, E. H. S. Acompanhamento da estabilidade oxidativa do biodiesel através da calorimetria exploratória diferencial pressurizada (PDSC) durante o processo de estocagem. 2007. Disponível em: <http://www.biodiesel.gov.br/docs/ congresso2007/armazenamento/8.pdf $>$. Acesso em: 15 jul. 2009.

CARNEIRO, P. I. B.; REDA, S. Y.; CARNEIRO, E. B. B. ${ }^{1} \mathrm{H}$ NMR characterization of seed oils from rangpur lime (Citrus limonia) and "sicilian" lemon (Citrus limon). Annals of Magnetic Resonance, Rio de Janeiro, v. 4, p. 64-68, 2005.

CARRASCO, F.; The evaluation of kinetic parameters from thermogravimetric data. Thermochimica Acta, v. 213, p. 115, 1993.

DANTAS, H. J. Estudo termoanalítico, cinético e reológico de biodiesel derivado do óleo de algodão (Gossypium hisutum). 2006. Dissertação (Mestrado em Química) - Universidade Federal da Paraíba, Paraíba.

FERRARI, R. A.; OLIVEIRA, V. S.; SCABIO, A. Biodiesel de soja: taxa de conversão em ésteres etílicos, caracterização físico-química e consumo em gerador de energia. Química Nova, São Paulo, v. 28, n. 1, p. 19-23, 2005.

FERRARI, R. A.; SOUZA, W. L. Avaliação da estabilidade oxidativa de biodiesel de óleo de girassol com antioxidantes. Química Nova, São Paulo, v. 32, n. 1, p. 106-111, 2009.

LIANG, Y. C.; MAY, C. Y.; FOON, C. S.; NGAN, M. A.; HOCK, C. C.; BARISON, Y. The effect of natural and synthetic antioxidants on the oxidative stability of palm diesel. Fuel, London, v. 85, n. 5/6, p. 867-870, 2006.

LIMA, L. N.; CARVALHO, M. W. N.; SANTOS, J. C. O. Caracterização Térmica e Oxidativa do Biodiesel Derivado do Óleo de Algodão. 2007. Disponível em: $<$ www.annq.org/congresso2007/trabalhos_apresentados/ T20.pdf> Acesso em: jul. 2009

MOTHÉ, C. G..; CORREIA, D. Z.; CASTRO, B. C. S.; CAITANO, M.; ARANDA, D. A. G.. Biodiesel obtido a partir de rejeito de gordura animal. In: CONGRESSO BRASILEIRO DE PLANTAS OLEAGINOSAS, ÓLEOS, GORDURAS E BIODIESEL, 2., 2005, Varginha. Anais... Varginha, 2005. p. 743-747.

OLIVEIRA, M. J. Transições de fase e fenômenos críticos. São Paulo: Instituto de Física Universidade de São Paulo, 2006. Disponível em: <http://fge.if.usp. br/ oliveira/tffc1.pdf>. Acesso em: 15 jul. 2009.

REDA, S. Y.; CARNEIRO, P. I. B. Parâmetros físicoquímicos do óleo de milho in natura e sob aquecimento calculado pelo programa PROTEUS RMN H1. Publicatio UEPG: Ciências Exatas e da Terra, Agrárias e Engenharias, v. 12, n. 2, p. 31-36, 2006.

REDA, S. Y.; COSTA, B.; FREITAS, R. J. S. Aplicação do programa JV.NH-1 na caracterização do biodisel etílico de semente de limão rosa (Citrus limonia O.), uma fonte não convencional. In: JORNADA BRASILEIRA DE RESSONÂNCIA MAGNÉTICA, 10., 2008, Niterói. Anais... Niterói, 2008a. p. 211.

Determinação da glicerina livre em amostras de biodiesel etílico por RMN de hidrogênio. Revista Analytica, Rio de Janeiro, v. 43, p. 86-90, 2008 b.

. Determinação do índice de acidez por $R M N-H^{1}$ do biodiesel etílico de milho. 2007a. Disponível em: <http://www.biodiesel.gov.br/docs/congresso2007/ caracterizacao/23.pdf>. Acesso em: 10 jan. 2010.

Determination of iodine value in ethylic biodiesel samples by 1H-NMR. Annals of Magnetic Resonance, Rio de Janeiro, v. 6, n. 3, p. 69-75, $2007 \mathrm{~b}$.

ROSENHAIM, R. Avaliação das propriedades fluidodinâmicas e estudo cinético por calorimetria exploratória diferencial pressurizada (PDSC) de biodiesel etílico derivado de óleo de fritura usado. 2009. Tese (Doutorado em Química) - Universidade Federal da Paraíba, Paraíba.

WENDHAUSEN, P. A. P. Análises térmicas. 2006. Disponível em: <www.materiais.ufsc.br/Disciplinas/ EMC5733/Apostila.pdf>. Acesso em: 15 jul. 2009.

REDA, S. Y. Estudo de Antioxidantes e o Seu Possivel Efeito Protetor em óleos Vegetais Sob Aquecimento. Rev. Ciência e Tecnologia de Alimentos. No prelo.

Recebido em 17 Maio, 2010 - Received on May 17, 2010 Aceito em 7 Setembro, 2010-Accepted on September 7, 2010. 